\author{
Mr. CHRISTIAN DÖRFLER, Lektor \\ WIFI Österreich
}

\title{
QUALITÄTSMANAGEMENT IM TOURISMUS
}

UDK: 338.48:65.012

Empfangen: 1. Juli 1996

Fachlicher Beitrag

\begin{abstract}
"Gäste beschweren sich nicht, sie kommen einfach nicht mehr"
Dienstleistungsqualităt und Qualitätsmanagement sind daher die wichtigsten Aspekte für die zukünftige europäische Tourismusentwicklung, sei es für eine Region oder den einzelnen Betrieb.

Voraussetzung um Qualitätsmanagement zu implementieren, ist die genaue Kenntnis der unternehmensspezifischen Prozesse und das Messen der Kundenzufriedenheit z.B. durch Gästebefragungen.

Die Erlebniswelt des Gastes muß in ihrer Gesamtheit - vom telefonischem Erstkontakt bis hin zum Aus-Checken - Beachtung finden. Daraus resultieren auch neue Aufgaben für Führungskräfte. Gefordert sind - neben Sachkompetenz - auch soziale Kompetenz, persönliche Kompetenz und Kompetenz für Methoden.

Die ISO 9000-Normenreihe ist ein Versuch Qualitätsmanagement in Betrieben anhand von Checklisten zu implementieren. Seriöse Beratung ist aber bei der Installierung von Qualitätsmanagementsystemen in jedem Fall zu empfehlen.
\end{abstract}

Schlüsselwörter: Dienstleistungsqualität, Qualitätsmanagement, Tourismus

\section{EINLEITUNG}

\section{„Gäste beschweren sich nicht - sie kommen einfach nicht mehr“ 1}

Wie in vielen anderen Dienstleistungsbereichen gewinnt die Erkenntnis über die Bedeutung von Kundenzufriedenheit auch im Tourismus immer mehr an Bedeutung. Die Gäste werden immer selbstbewußter und haben aufgrund der erhöhten Reisetätigkeit auch immer bessere Vergleichsmöglichkeiten zwischen unterschiedlichen Angeboten. Gleichzeitig erkennt man aus Umfragen, daß das Beschwerdeverhalten relativ schwach ausgeprägt ist. Dies bedeutet, daß viele Unternehmer gar nicht über die Zufriedenheit ihrer Gäste Bescheid wissen. Aus diesem Grund ist es ein Gebot der Zeit auch in Tourismusbetrieben Qualitätssicherungssystems einzuführen, da ein alleiniges Reagieren auf Reklamationen und Beschwerden möglicherweise zu spät - sicher aber unzureichend ist.

1 Kohl, „Qualität im Tourismus“, S. 10, Wirtschaftsverlag 


\section{QUALITÄT IM WANDEL DER ZEIT}

Es wäre sicher interessant, die heutigen Kundenwünsche in bezug auf touristische Qualität mit jenen unserer Vorfahren zu vergleichen. Noch in der Nachkriegszeit war man froh, überhaupt eine Unterkunft für die Ferien - so man sich solche leisten konnte zu bekommen. Heute, wo das touristische Angebot für breite Bevölkerungsschichten immer vielfältiger wird, steigen auch die Ansprüche. Zweifellos ist es daher so, daß für die Anbieter touristischer Leistungen die Anforderungen immer größer werden. Der Gast von heute erwartet sich eine genau auf ihn zugeschnittene gleichbleibende Leistung. Dies bedeutet zweierlei:

1.) Die Zielgruppen müssen genau analysiert und individuell angesprochen werden;

2.) Die Qualität der erbrachten Dienstleistung muß dauerhaft gewährleistet werden können.

Speziell im letzten Jahrzehnt wurde im österreichischen Tourismus der Begriff „Qualität" primär mit:

- der Anzahl der Sterne oder

- dem Vorhandensein von Hallenbädern, Tennisplätzen, Liftanlagen etc.

gemessen. D. h. es wurde in erster Linie in die Ausstattung (touristische Hardware) investiert. Dieser Schritt in die sicherlich richtige Richtung auf dem Weg zum Qualitätstourismus hatte jedoch u.a. zwei Effekte:

1.) Die Verschuldung der Branche stieg rasant an;

2.) Die Verbesserung der Dienstleistungsqualität (Software) konnte nicht mitwachsen bzw. verschlechterte sich sogar teilweise;

Gleichzeitig sind die Konsumenten aber immer preissensibler geworden. Man ist bereit, für entsprechende (genau die richtige) Leistung gutes Geld zu bezahlen, erkennt aber auch sehr genau, wenn das Preis-Leistungsverhältnis unausgewogen ist.

\section{PREIS- ODER QUALITÄTSWETTBEWERB?}

Trotz der wirtschaftlich ohnehin bereits sehr angespannten Situation, führen viele Betriebe den Wettbewerb um alte bzw. neue Kundensegmente über den Preis. Dies beginnt bei Sonderrabatten für Spezialtermine und endet bei 7 Tage wohnen, 5 Tage zahlen. Nebenbei versuchen viele Gemeinden bzw. Betriebe mit all-inklusive-Angeboten den Gast zu ködern.

Aus betriebswirtschaftlicher Sicht kann der reine Preiswettbewerb nicht begrüßt werden. In Mitteleuropa - speziell aber in Österreich, Deutschland und der Schweiz sind die Umfeldbedingungen für den Tourismus einfach andere als in Dritte-Welt-Staaten oder Weichwährungsländern. Umweltauflagen, Personalkosten etc. machen es unmöglich den Preiswettbewerb zu gewinnen. Auch aus preispsychologischer Sicht ist ein zu großes bzw. schnelles Nachgeben beim Preis nicht sinnvoll. Ein jahrelang mühsam aufgebautes Preisniveau kann durch „Schleuderaktionen“ innerhalb einer Woche zerstört werden. 


\section{Warum Firmen Kunden verlieren ...}

Untersuchungen darüber - warum Dienstleistungsfirmen Kunden verlieren zeigen, daß $68 \%$ der Kunden die Firma wechseln, weil sie sich nicht gut behandelt fühlten, nur $9 \%$ der Kunden hingegen gingen verloren, weil andere Angebote billiger waren. Auch in einem "Spiegel"-Artikel vom Vorjahr werden unter dem Titel "Störenfried Kunde - vom König zum Bittsteller" 25 \% Umsatzverlust durch schlechte Dienstleistung für möglich gehalten. ${ }^{2}$ Auf die Frage: „Was stört Sie sehr ?" antworten 72 $\%$ der Konsumenten mit "unfreundliches Personal“ und $61 \%$ mit "mangelnde Hilfsbereitschaft".

\begin{tabular}{|c|c|}
\hline \multicolumn{2}{|c|}{ Warum verlieren Firmen Kunden? } \\
\hline$(-)$ & $1 \%$ stribt \\
\hline$\oplus \odot \oplus$ & $3 \%$ ziehen um \\
\hline$\oplus \odot \oplus \odot)$ & 4\% lieben Abwechslung \\
\hline 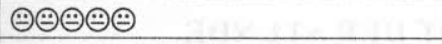 & 5\% wechseln auf Empfehlung von Freunden \\
\hline 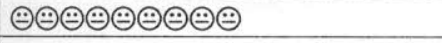 & $9 \%$ finden andere Leistungen preiswerter \\
\hline$\odot \odot \oplus \odot \oplus \odot \odot \odot \odot \odot \odot \odot \odot$ & $10 \%$ sind notorische Querulanten \\
\hline 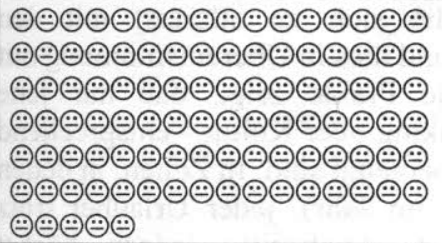 & $68 \%$ fühlen sich nicht gut behandelt \\
\hline
\end{tabular}

Quelle: PDC-Kundenzufriedenheitsforschung 1995

\section{Beschwerdeverhalten von Kunden}

Wie bereits eingangs erwähnt, beschweren sich viele unserer Gäste nicht. Sie kommen einfach nicht mehr. Der Grund dafür ist sicher auch im schlecht ausgeprägten Kommunikationsverhalten unseren Gästen gegenüber zu begründen. Kritik wird vielfach nicht als Chance erkannt, sondern der kritisierende als Querulant abgetan.

Aus einer Untersuchung der "SWISSAIR" weiß man, daß nur $4 \%$ der Unzufriedenen sich beschweren. Dies bedeutet: $96 \%$ der vom Gast wahrgenommenen Fehler werden nicht bekannt, daher können auch $96 \%$ der Chancen etwas im Betrieb zu verbessern, nicht wahrgenommen werden.

Aus anderen Untersuchungen geht hervor, daß jeder unzufriedene Gast mindestens 10 Personen, $13 \%$ der unzufriedenen Gäste sogar 20 Personen aus ihrem Bekanntenkreis über unangenehme Erlebnisse auf Reise informieren.

Daraus ergibt sich folgende Rechnung: Erhält ein Hotel 50 Beschwerden im Jahr, so entspricht dies in etwa 1.250 unzufriedenen Gästen. Wenn jeder dieser unzufriedenen Gäste nur 10 Personen aus dem Bekanntenkreis informiert, so sind dies 12.500 Personen, welche über einen Fehler in einem bestimmten Hotel informiert wurden. 
In einem 100-Betten-Hotel gibt es durchschnittlich pro Tag 1.000 Kontakte zwischen den Gästen und der Hotelcrew. Jeder dieser Gästekontakte ist der „Augenblick der Wahrheit" für die zukünftige emotionale Bindung des Gastes an den Betrieb. Bei jedem dieser kurzen Kontakte wird entschieden, ob der Gast die Dienstleistung als positiv oder unangenehm erlebt. ${ }^{3}$

Gäste objektivieren jedoch nicht die Gesamtleistung. Ist von 5 Gästekontakten einer negativ, so wird die Gesamtleistung als negativ betrachtet. Es ist für das Management daher von immenser Bedeutung, durch Qualitätsmanagementsysteme die Wahrscheinlichkeit von Fehlleistungen zu minimieren. Es muß die gesamte Leistungskette funktionieren. Die Leistung muß homogen sein. Wettbewerb primär über den Preis zu führen kann in Mitteleuropa daher keine sinnvolle Strategie für den Tourismus sein.

\section{QUALITÄTSMANAGEMENT - EIN GEBOT DER STUNDE}

Qualität können nur jene Betriebe liefern, die sich zu allererst mit den Erwartungen und Ansprüchen ihrer Gäste beschäftigen und dies in Übereinstimmung mit dem persönlichen Unternehmensleitbild bringen. Die Praxis zeigt, daß nur jene Unternehmer ihre Spezial-angebote - sei es Mountainbiking oder Kultur - entsprechend den Gästen verkaufen können, die selbst von der Idee überzeugt sind. In Zeiten, in denen die Ansprüche an den Urlaub steigen (schönste Zeit im Jahr), jeder Urlauber trotz Massentourismus zunehmende Individualität verlangt gleichzeitig jedoch höchst sensibel das Preis-Leistungsverhältnis betrachtet, ist höchste Professionalität in der Dienstleistung gefragt.

\section{Die neue professionelle Dienstleistungsqualität}

In vielen anderen Branchen hat man es bereits erkannt. Nun greift dieser Trend auch auf die Tourismusindustrie über. Nur professionell organisierte Abläufe - in Verbindung mit auf diese Abläufe ausgebildetem Personal - gewährleisten Qualität gegenüber dem Gast. In den großen Konzernen arbeitet man schon seit langem mit Qualitätshandbüchern und Standards. In der europäischen Klein- und Mittelbetriebsstruktur glauben viele aber immer noch Qualität dem Zufall überlassen zu können. Selbst einfache Checklisten werden als „Erfindung wirklichkeitsfremder Bürokraten" abgetan.

Qualität ist ein zu komplexes Thema um sie dem Zufall zu überlassen.

3 Dörfler, Taschenfolder „Gedanken zum Thema Gastlichkeit" 


\section{Die Erlebniswelt der Kunden}

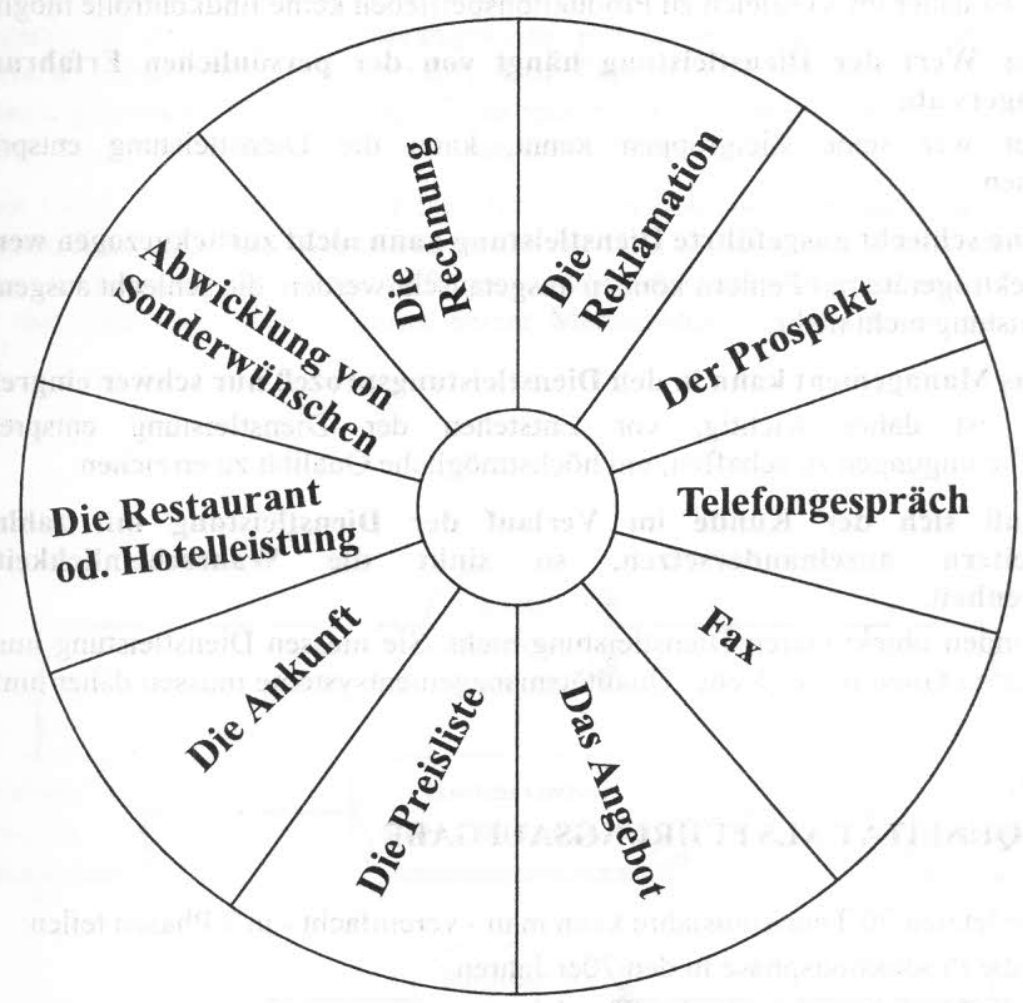

Jede der im „Qualitätskreis“ angeführten Schnittstellen zwischen Hotelcrew und Gast stellt einen Meilenstein in Richtung „Qualitätserlebnis“ des Gastes dar. Ob die gewünschte Qualität erreicht wird hängt primär von der Unternehmensführung $a b$. In jenen Betrieben, in denen die Zusammenhänge zwischen Buchungsverhalten und echter Dienstleistungsqualität erkannt wurden, werden auch die Mitarbeiter entsprechend eingebunden werden und das Qualitätssicherungssystem laufend wachsen.

Professionelle Dienstleistungsqualität orientiert sich nicht am Mittelmaß, sondern an betriebsindividueller, zielgruppenorientierter Spitzenleistung. Mittelmäßige Leistungen stellen den Gast nicht zufrieden. Unzufriedene Gäste denken über den Preis nach bzw. kommen nicht wieder. Viele Betriebe, investieren heute ihr Geld noch immer lieber in Werbemaßnahmen als in Qualitätssicherungssysteme. Dies obwohl bekannt ist, $\mathrm{da} ß$ es $10 \mathrm{Mal}$ soviel kostet einen neuen Gast zu gewinnen, als einen bestehenden zu halten.

\section{Right first time}

Bei Dienstleistungsbetrieben ist es im Vergleich zu Produktionsbetrieben besonders schwierig "Qualitätssicherung“ zu betreiben. Folgende Gründe sind dafür ausschlaggebend: 


\section{Dienstleistung entsteht zum Zeitpunkt der Lieferung.}

Es ist daher im Vergleich zu Produktionsbetrieben keine Endkontrolle möglich.

Der Wert der Dienstleistung hängt von der persönlichen Erfahrung des Empfängers ab.

Nur wer seine Zielgruppen kennt, kann die Dienstleistung entsprechend abstimmen.

\section{Eine schlecht ausgeführte Dienstleistung kann nicht zurückgezogen werden.}

Elektrogeräte mit Fehlern können ausgetauscht werden, die schlecht ausgetauschte Dienstleistung nicht mehr.

Das Management kann in den Dienstleistungsprozeß nur schwer eingreifen.

Es ist daher wichtig, vor Entstehen der Dienstleistung entsprechende Rahmenbedingungen zu schaffen, um höchstmögliche Qualität zu erreichen.

Muß sich der Kunde im Verlauf der Dienstleistung mit zahlreichen Mitarbeitern auseinandersetzen, so sinkt die Wahrscheinlichkeit der Zufriedenheit.

Kunden objektivieren Dienstleistung nicht. Sie messen Dienstleistung immer am schwächsten Glied in der Kette. Qualitätsmanagementsysteme müssen daher umfassend sein!

\section{QUALITÄT ALS FÜHRUNGSAUFGABE}

Die letzten 20 Tourismusjahre kann man - vereinfacht - in 3 Phasen teilen:

- die Produktionsphase in den 70er Jahren

- die Marketingphase in den 80er Jahren

- die Qualitätsphase im ausgehenden 20 Jhd.

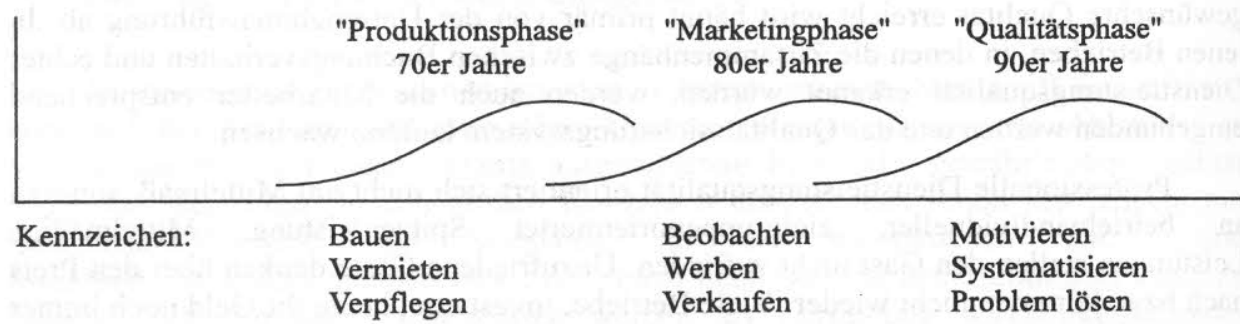

In der ersten Zeit der touristischen Aufbauphase mit enormen Wachstumsraten wurden viele Betriebe neu gebaut und reines Absatzmarketing betrieben. Die Nachfrage war größer als das Angebot. In der zweiten Phase - einer Zeit stagnierender bzw. nur leicht wachsender Nachfrage - wurde durch Marktforschung und Marktbeobachtung stärker auf den Gast eingegangen und so die Nachfrage erhöht. Nun - in der dritten Phase - hat man erkannt, daß der Gast die einzig bestimmende Größe des Marketing ist und daß die Treue zu einem Betrieb sehr stark auf dem individuellen Qualitätserlebnis beruht. Jene Betriebe, die sich dieser Herausforderung nicht rasch stellen, werden vom Markt verschwinden. ${ }^{4}$ 
Diese Erkenntnis bedeutet aber auch, daß in Zukunft die Erfolgsfaktoren weniger in der Ausstattung (Hardware) zu suchen sein werden, sondern vielmehr im Führungsstil, in der Art der Umgangsweise mit Gästen und Mitarbeitern und im konzeptiven Durchdenken und schriftlichen Festlegen (Software) von typischen Situationen gegenüber dem Gast. Qualitätsdenken entsteht jedoch nicht von allein. Es muß vom Management gelebt und vor allem ermöglicht werden.

Die Chefs der Vergangenheit beriefen sich primär auf ihre Sachkompetenz und haben übersehen, daß ihre Macht von heute auf dem Wissen von gestern beruht. Der Manager von heute ist nur mehr zu $50 \%$ ein Fachmann, aber zu weiteren $50 \%$ Coach, Trainer und Motivator seines Teams, seiner Mannschaft - er ist Leader und Teil des Teams. $^{5}$

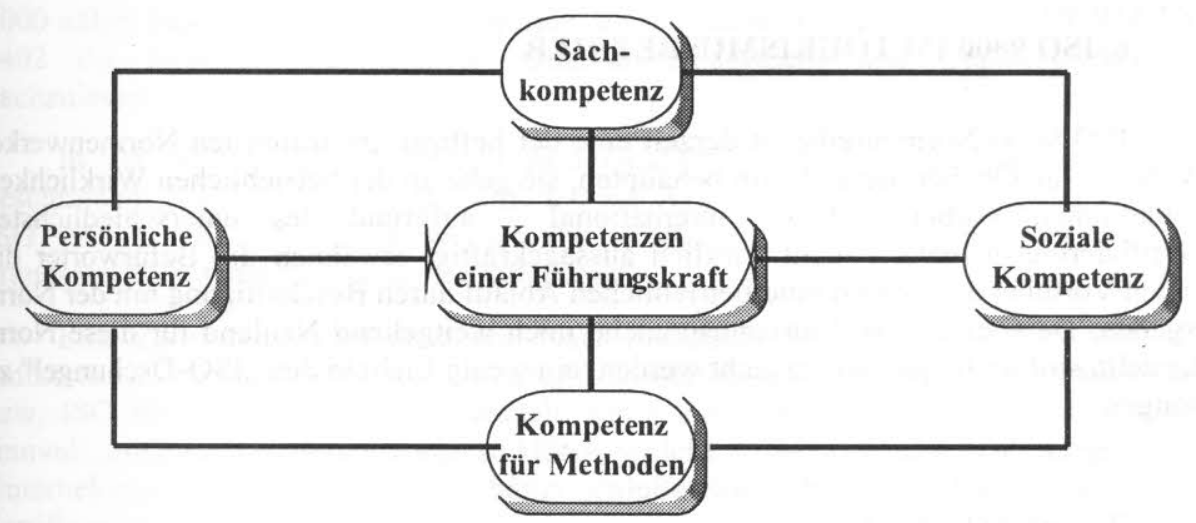

\section{DIE IMPLEMENTIERUNG VON QUALITÄTSMANAGEMENT - SYSTEMEN}

In Produktionsbetrieben haben div. Konzepte der Qualitätssicherung bereits seit langem Einzug gehalten. Im Tourismus ist die Übernahme dieser Systeme - ohne entsprechende Adaption - aber schwierig.

Da das Qualitätsmanagement sehr stark von der Unternehmenskultur abhängt, können Qualitätssicherungssysteme erfahrungsgemäß nur schrittweise implementiert werden.

4. Dörfler,Kohl. Siegl, ,1000 Jahre Gastlichkeit - Qualität im Tourismus“ S. 17, WIFI Schriftenreihe

5 Dörfler, Kohl, Siegl, „1000 Jahre Gastlichkeit - Qualität im Tourismus” S.24, WIFI Schriftenreihe 


\section{Die vier Stufen des Qualitätsmanagements im Tourismus:}

1. Durch regelmäßige Mitarbeiterbesprechungen und Schulungen wird versucht, möglichst hohe und konstante Dienstleistungsqualität zu erreichen.

2. Innerbetriebliche Qualitätsstandards werden erarbeitet und schriftlich bzw. visuell festgehalten. Auf Basis eines Unternehmensleitbildes werden die meisten Entscheidungen im Unternehmen getroffen.

3. Es wird - auf Basis der international gültigen ISO 9000-Qualitätsstandards ein Qualitätshandbuch erstellt. Das Qualitätssystem wird durch ein unabhängiges Institut überprüft (Zertifizierung).

4. $\mathrm{TQM}=$ Total Quality Management; Das wesentliche Element von TQM ist eine durchgehende Einstellungsänderung im Sinne von Prozeßverantwortung, vorbeugenden Handeln und der Selbstverpflichtung fortlaufend besser zu werden um somit höchste Dienstleistungsqualität zu erreichen.

\section{ISO 9000 IM TOURISMUSBETRIEB}

ISO 9000-Normenreihe ist derzeit eine der heftigst umstrittensten Normenwerke. Während die Gegner dieser Norm behaupten, sie gehe an der betrieblichen Wirklichkeit vollkommen vorbei und sei international - aufgrund des unterschiedlichsten Zertifizierungsniveaus - nicht wirklich aussagekräftig, erwähnen die Befürworter die vielen Vorteile, die sich im innerbetrieblichen Ablauf durch Beschäftigung mit der Norm ergeben. Da speziell die Tourismusbranche noch weitgehend Neuland für diese Norm darstellt, soll im folgenden versucht werden, ein wenig Licht in den „ISO-Dschungel“ zu bringen.

\section{Was ist ISO $9000 ?$}

Eine Checkliste, ob ein Unternehmen gut organisiert ist. Anhand von 20 Kapiteln (Nachweisstufe 9001) werden die unterschiedlichsten betrieblichen Abläufe/Themen untersucht und im Qualitätshandbuch festgehalten.

Wie zum Beispiel:

- Hat das Unternehmen überhaupt ein konkretes Ziel ?

- Sind alle Abläufe und deren Stärken und Schwächen bekannt?

- Was sind die Wünsche der Gäste?

- Wie sind die täglichen Arbeiten organisiert?

Oft wird die Frage gestellt, wie sich eine Zertifizierung vieler Betriebe auf die österreichische Tourismuswirtschaft auswirken würde. Der Hintergrund dieser Fragestellung ist die Angst vor einer Vereinheitlichung der österreichischen Tourismuslandschaft. Ein Unternehmer fragte z.B.: „Warum soll der Gast zu mir kommen, wenn in meinem Unternehmen das Service genau gleich wie bei der Konkurrenz ist?" Die Befürchtung ist verständlich, kennt doch jeder technische Normen. Diese technischen Normen haben die Eigenschaft, gewisse Gegenstände möglichst genau zu definieren. Dies erleichtert es Produkte unterschiedlicher Firmen miteinander 
zu kombinieren. So ist es z.B. selbstverständlich, daß das Gewinde der Glühbirne exakt in die Fassung paßt. Technische Normen geben gewöhnlich keinerlei Möglichkeiten zu Individualismus. Im Gegensatz dazu legt das ISO 9000-Normenmodell lediglich die Kriterien fest, nach welchen die Abläufe und Qualitätsrichtlinien eines Betriebes analysiert und niedergeschrieben werden müssen. Die Inhalte selbst werden jedoch nicht normiert. Die Befürchtung, daß durch Qualitätsmanagement die Individualität des Betriebes bzw. des Tourismus verloren geht, trifft also nicht zu. Im Gegenteil, ein Qualitätsmanagement-system hilft dem einzelnen Unternehmer die Individualität der Dienstleistung genau festzulegen und sich so dauerhaft von seinen Mitbewerbern abzugrenzen.

\section{Der Aufbau der ISO 9000-Normenreihe}

Wenn man sich mit dem Aufbau eines Qualitätsmanagementsystems nach ISO 9000 näher beschäftigen will, so empfiehlt es sich zunächst einmal in der NORM ISO 8402 die Begriffe zum Thema "Qualitätsmanagement und Qualitätssicherung“ nachzulesen.

In der NORM EN ISO 9000 sind Qualitäts- und Qualitätssicherungsnormen beschrieben. Diese Norm ist ein Leitfaden zur Auswahl und Anwendung der Normen ISO 9001 - ISO 9003. Hier werden die wesentlichen Unterschiede zwischen den grundsätzlichen Qualitätskonzepten aufgezeigt.

Eine Zertifizierung ist nach drei Nachweisstufen möglich, wobei ISO 9001 die umfassendste darstellt. Dies bedeutet aber keinesfalls, daß Betriebe, die nach ISO 9002 bzw. ISO 9003 zertifiziert sind, deshalb schlechtere Qualität anbieten. Es ist daher sinnvoll, mit einem Experten vorab zu klären, welche Arbeitsbereiche bzw. Aufgaben im Unternehmen anfallen, denn davon hängt schlußendlich ab, welche Nachweisstufe zur Zertifizierung herangezogen wird.

Derzeit geht die Tendenz bei Tourismusbetrieben im deutschsprachigen Raum in Richtung Zertifizierung nach ISO 9001, welche sich von ISO 9002 im wesentlichen dadurch unterscheidet, daß hier auch die Entwicklung neuer Angebote mitberücksichtigt wird. Wenn z.B. ein Seminarhotel zum Wochenende öfters Packages für unterschiedliche Zielgruppen anbietet (z.B. ein Seniorenwochende mit Theaterbesuch und Fiakerfahrt) so stellt dies die Entwicklung eines touristischen Angebots dar und der Betrieb sollte nach ISO 9001 zertifizieren.

Die NORM ISO 9004 ist ein Leitfaden zur Erläuterung bei der Einführung eines Qualitätmanagementsystems. Besonders erwähnenswert ist für den Tourismus dabei 9004/2, der Leitfaden zur Einführung eines Qualitätsmanagementsystems bei Dienstleistungsunternehmen. 
Aufbau der ISO 9000-Normenreihe

ISO 8402

or Begriffe/Definitionen

ISO 9000 Teil 1 or Auswahlkriterien für die, ,richtige“ Nachweisstufe

Nachweisstufen

ISO 9001 Go Entwicklung/Design

ISO 9002 or Produktion

or Montage

or Wartung / Kundendienst

ISO 9003 as Endprüfung

ISO 9004 Teil 1 Go Leitfaden für Entwicklung des individuellen QM-Systems

ISO 9004 Teil 2 or Leifaden für Dienstleistungsunternehmen

\section{Beispiel aus einem Qualitätshandbuch}

Die - im Zuge der Erarbeitung des Qualitätshandbuches als wesentlich erkannten Abläufe, werden in sogenannten "Verfahrensanweisungen“ entweder verbal oder grafisch dargestellt und bilden einen Teil des Qualitätshandbuches. Dies trägt wesentlich zur Einhaltung eines gleichbleibenden Qualitäsniveaus bei. Im unten stehenden Beispiel wurde in einem Betrieb der Ablauf bei schriftlichen Anfragen festgelegt. 


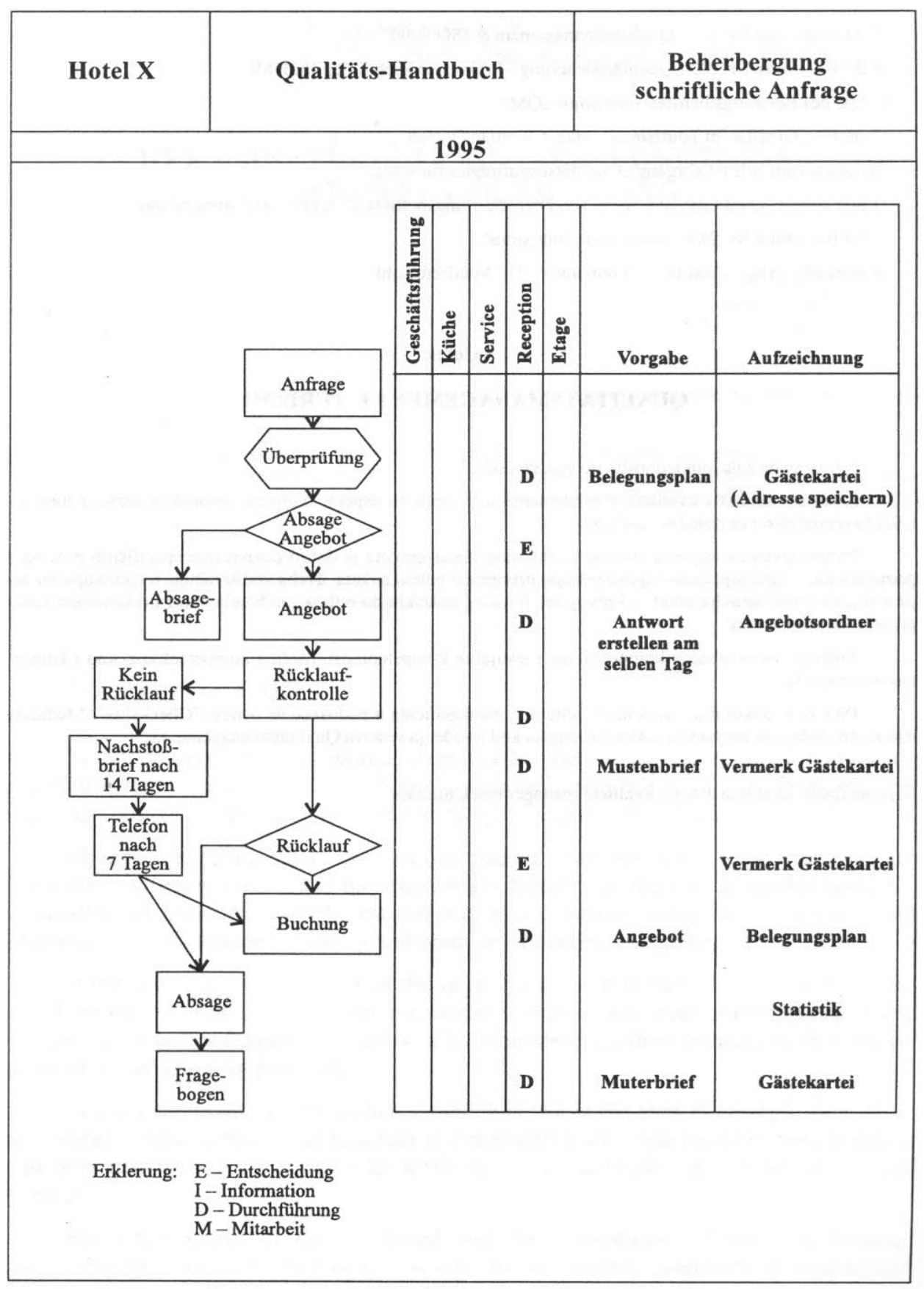




\section{LITERATURLISTE}

- WIFI Broschüre Nr. 251 „Qualitätsmanagement \& ISO 9000“

- WIFI Broschüre Nr. 213 „Qualitătssicherung - Praktische Richtlinien für KMU“

- Folder des Beratungsdienstes Tourismus "QM“

- Skriptum „Qualität im Tourismus“, Mag. Christian Dörfler

- Skriptum vom WIFI-Lehrgang „Qualitätsbeauftragter für KMU“

- Diplomarbeit „Qualitätsmangement von Dienstleistungsbetrieben“, Christian Zimmermann

- WIFI Broschüre Nr. 285 „Qualität im Tourismus“,

- Wirtschaftsverlag „Qualităt im Tourismus“, Dr. Manfred Kohl

\section{Sažetak}

\section{QUALITÄTSMANAGEMENT U TURIZMU}

"Gosti se ne žale, oni jednostavno više ne dođu."

Kvaliteta usluga i kvaliteta managementa najvažniji su aspekti budućeg europskog razvoja turizma, kako za regiju tako i za pojedino poduzeće.

Pretpostavka za implementiranje kvalitetnog managementa je dobro poznavanje specificnih procesa u poduzeću kao i mjerenje zadovoljstva gostiju, primjerice putem anketa. Treba voditi računa o cjelokupnom dojmu koji na gosta ostavlja hotel: od prvog telefonskog kontakta do odlaska iz hotela, što za rukovodeći kadar predstavlja novi izazov.

Traži se, osim stručne kompetencije, i socijalna kompetentnost, osobna kompetentnost, kao i kompetentnost metoda.

ISO 9000 pokušaj je uvođenja kvalitetnog managementa u poduzeće na osnovi "Checkslita". Međutim, u svakom slučaju se preporuča savjet stručnjaka kod uvođenja sustava Qualitătsmanagementa.

Ključne riječi: kvaliteta usluga, kvaliteta managementa, turizam 